\title{
Capacidades y retos del monitoreo sobre la disponibilidad y el acceso a los servicios de salud en Argentina
}

\author{
Capabilities and challenges in monitoring the \\ availability of and access to health services in \\ Argentina
}

${ }^{1}$ Doctor en Demografía. Investigador Asistente, Consejo de Investigaciones Científicas y Técnicas (CONICET), Centro de Investigaciones y Estudios sobre Cultura y Sociedad (CIECS), Córdoba, Argentina. $\square$ (iD)

\section{Andrés Conrado Peranovich ${ }^{1}$}

RESUMEN El monitoreo de la utilización, calidad y rendimiento de los servicios de salud, a través de diversos indicadores, es una exigencia creciente para quienes toman decisiones en el tema. Teniendo en cuenta la importancia de contar con indicadores de disponibilidad, calidad y acceso a los servicios de salud por parte de la población, el objetivo de este artículo es investigar la capacidad de las fuentes de información producidas oficialmente en Argentina, desde el año 2000, de generar estos indicadores, identificando además en qué medida dichas fuentes cumplen con los requisitos de periodicidad, desagregación y publicación. Según las pesquisas realizadas, la información disponible a nivel oficial en el país no permite calcular la totalidad de indicadores o solo pueden ser construidos para un momento en el tiempo, lo que evidencia la necesidad de incorporar nuevas fuentes, o bien adaptar las existentes.

PALABRAS CLAVES Fuentes de Datos; Servicios de Salud; Salud Pública; Accesibilidad a los Servicios de Salud; Argentina.

\begin{abstract}
Monitoring the utilization, quality and performance of health services through indicators is a growing necessity of decision makers. Given the importance of indicators related to availability of, quality of and access to health services by the population, the objective of this article is to research the capacity of information sources produced officially in Argentina since 2000 to generate such indicators, and to identify the extent that such sources comply with the requirements of frequency, disaggregation and publication. The research results show that the information officially available in the country does not allow for the calculation of all indicators, or allows for their construction at only some moments in time, evidencing the need to incorporate new sources or adapt existing ones.
\end{abstract}

KEY WORDS Data Sources; Health Services; Public Health; Health Services Accessibility; Argentina. 


\section{INTRODUCCIÓN}

Dada la importante repercusión que tienen las enfermedades en el crecimiento económico de las sociedades, invertir en los sistemas de salud constituye un componente substancial de las estrategias generales de desarrollo ${ }^{(1)}$. Esta inversión debe ser eficaz y sustentable, por lo que debe estar fundamentada en datos precisos de la realidad.

Por ello, el monitoreo de la utilización, calidad y rendimiento de los servicios de salud a través de indicadores específicos se considera fundamental para el progreso de los sistemas de salud, y una exigencia creciente para quienes toman decisiones en el tema. Estos indicadores también son útiles para la programación local, provincial y nacional y, complementariamente, para estudios e investigaciones especiales y para el cálculo del costo de la atención en salud.

$\mathrm{Al}$ igual que en otras áreas de salud, el monitoreo de los servicios de salud requiere la generación de herramientas que cumplan, al menos, con los siguientes requisitos: periodicidad (para detectar fortalezas, debilidades y progresos en el tiempo); desagregación (para identificar los sectores/grupos poblacionales más desfavorecidos en cuanto al acceso a tales servicios); y publicación (en función del derecho que tiene la comunidad de Conocer la situación de estos servicios) ${ }^{(2)}$.

Teniendo en cuenta la importancia de contar con indicadores de disponibilidad, calidad y acceso a los servicios de salud por parte de la población, el objetivo de este trabajo es investigar la capacidad de las fuentes oficiales de información en Argentina, desde el año 2000, para generar tales indicadores, identificando además en qué medida dichas fuentes cumplen con los requisitos referidos.

\section{METODOLOGÍA}

En primer lugar, se definen las áreas temáticas sobre las que se van a construir los indicadores referidos al monitoreo de los servicios de salud: utilización, disponibilidad, cobertura, acceso y calidad.

La utilización de los servicios de salud se refiere al uso que hacen los individuos de la infraestructura o de los establecimientos, bienes y servicios de salud. La disponibilidad se mide a través de los recursos humanos, materiales y de infraestructura para dar atención a los problemas de salud de la población en relación con la población que el Estado está potencialmente obligado a atender. Se ubican aquí los indicadores relacionados con la oferta de hospitales, clínicas, consultorios, camas, medicamentos y personal médico, destinados a la prestación de servicios de salud. Ambas áreas, utilización y disponibilidad, corresponden a los conceptos de demanda y oferta que son utilizados como marco de estudio del proceso dinámico y complejo que pone en contacto a la población y el personal de salud, con el propósito de satisfacer una condición de salud determinada.

Los indicadores de cobertura de los servicios de salud reflejan en qué medida las personas que lo necesitan reciben efectivamente importantes intervenciones de salud ${ }^{(3)}$. Tales indicadores incluyen la atención a mujeres durante el embarazo y el parto, la inmunización para prevenir las infecciones más comunes de la infancia, la administración de suplementos de vitamina A en niños, y el tratamiento de las enfermedades más comunes de la infancia y las enfermedades infecciosas en adultos ${ }^{(3,4)}$.

El acceso a los servicios de salud se refiere a la posibilidad de obtener atención en salud que sea independiente de características como el ingreso, el lugar de residencia, la condición laboral o la pertenencia a un grupo étnico o minoría. Implica la eliminación de barreras u obstáculos, ya sean de tipo físico, económico, social o de información para el uso de los servicios de salud. De acuerdo con la Organización Mundial de la Salud (OMS), el acceso a los servicios de salud puede medirse a través de la utilización de los servicios de salud por parte de los individuos, la cual, a su vez, es influida por la estructura del sistema de servicios (disponibilidad de servicios, organización y financiamiento) y por 
la naturaleza de los requerimientos (recursos para los consumidores potenciales) ${ }^{(5)}$.

El ámbito de la calidad de los servicios de salud se refiere a que la atención e infraestructura sean adecuadas para las necesidades de salud de los pacientes, lo cual requiere, entre otros aspectos, de personal suficiente, medicamentos y equipamiento apropiados y suficientes, condiciones sanitarias y de higiene adecuadas, y buen trato por parte del personal de salud hacia las y los beneficiarios ${ }^{(6)}$.

Se procedió a la búsqueda y selección de indicadores relacionados con el monitoreo de la cobertura, disponibilidad, utilización, acceso y calidad de los servicios de salud, validados tanto en el ámbito internacional como nacional. A tales efectos, se relevaron los indicadores utilizados por organismos internacionales, como la Organización Mundial de la Salud, la Organización Panamericana de la Salud, el Banco Mundial, el Fondo de las Naciones Unidas para la Infancia, la Organización Internacional del Trabajo y la Comisión Económica para América Latina, y aquellos empleados a nivel nacional y en otros países de la región de las Américas.

Se estableció como criterio de selección: 1) la utilidad del indicador para la gestión de servicios de salud, en el sentido de su importancia para la evaluación de las distintas áreas de estudio; 2) su reconocimiento internacional, es decir que su uso sea difundido, permita realizar comparaciones con otras regiones y se adapte a la realidad de los sistemas de información de la región.

Posteriormente, se realizó el análisis de los cuestionarios y resultados provenientes de las fuentes de datos oficiales para evaluar la posibilidad de elaborar los indicadores seleccionados. A tal fin, se indagaron las siguientes fuentes oficiales producidas desde del año 2000, que contienen información relativa a la salud: Encuesta de Condiciones de Vida (ECV) 2001; Encuesta Nacional de Discapacidad (ENDI) 2002-2003; Encuesta Nacional de Nutrición y Salud (ENNyS) 2004-2005; Encuesta sobre Factores de riesgo (ENFR) 2005 y 2009; Encuesta de Utilización y Gasto en Servicios de Salud 2003, 2005 y 2010; Encuesta Nacional de Gastos de Hogares (ENGHo) 2004-2005 y 2012-2013; Encuesta Nacional sobre Calidad de Vida de Adultos Mayores (ENCaVIAM) 2012; Encuesta sobre Salud Sexual y Reproductiva (ESSyR) 2013; así como la información estadística procedente de los registros permanentes de la Dirección de Estadísticas e Información de Salud (DEIS) y demás institutos adheridos al Sistema Estadístico de Salud (SES) del ámbito del Ministerio de Salud de la Nación. Además, se contempló información producida al interior de la Dirección de Estadísticas a nivel provincial y datos provenientes del Censo Nacional de Población y Vivienda 2001 y 2010.

Finalmente, se examinó la capacidad que ofrecen las instituciones para elaborar los indicadores seleccionados según periodicidad, desagregación (geográfica, etaria, nivel socioeconómico, condición étnica y condición de discapacidad) y publicación.

\section{RESULTADOS}

Con base en la bibliografía y los sistemas de información de índole nacional e internacional, se seleccionan una serie de indicadores representativos de cada área temática de interés en servicios de salud, según criterios mencionados en el apartado metodológico. De esta forma se presentan 24 indicadores, catalogados en la Tabla 1 según área temática, definición e institución donde se encontró la referencia al indicador.

Esta lista de indicadores ofrece una guía resumida que permitiría caracterizar al sistema de servicios de salud de un territorio, en este caso, Argentina. Es decir, se trata de la mínima cantidad de indicadores con las que debería contar Argentina para evaluar el desempeño de su sistema de servicios de salud. Al respecto, la Tabla 2 muestra la posibilidad de aplicación de esta serie de indicadores a la totalidad del territorio argentino, la disponibilidad de datos por año y la fuente de origen de esos datos.

En la Tabla 2 se observa que 17 de los 24 indicadores pueden calcularse para toda 
Tabla 1. Denominación, definición e instituciones de referencia de indicadores seleccionados para monitorear la utilización, la disponibilidad, el acceso y la cobertura y la calidad de la atención de servicios de salud. Argentina, 2016.

\begin{tabular}{|c|c|c|c|}
\hline \multirow{2}{*}{$\begin{array}{l}\text { Área } \\
\text { temática }\end{array}$} & \multicolumn{3}{|c|}{ Indicadores seleccionados } \\
\hline & Denominación & Definición & Institución \\
\hline
\end{tabular}

Giro de cama

Estancia promedio

Atenciones por urgencia por

habitante
Razón de egresos hospitalarios
Número de atenciones ambulatorias de salud por cada 1.000 habitantes en una población, para un año dado, en un determinado país, territorio o área geográfica.

Número de mujeres gestantes que han recibido al menos una consulta de atención de salud durante el embarazo provista por un profesional o trabajador de la salud entrenado, expresado como porcentaje de la población de nacidos vivos, para un año dado, en un determinado país, territorio o área geográfica.

Número de egresos hospitalarios por cada 1.000 habitantes en una población, para un año dado, en un determinado país, territorio o área geográfica.

Es el número promedio de camas que estuvieron ocupadas diariamente durante un período, expresado en porcentaje.

SES

Es la rotación media de las camas y expresa cuántos pacientes pasan en un período dado, en promedio, por cama disponible.

Número promedio de días de atención prestada a cada paciente egresado durante el período.

Número de atenciones por urgencia de salud por cada 1.000 habitantes en una población, para un año dado, en un determinado país, territorio o área geográfica.

OPS

OPS

OPS SES SES OPS
Es el número de establecimientos de salud en determinada área geográfica OPS y/o dependencia administrativa en un período dado, por cada 1.000 habitantes de dicha área geográfica.

Número de camas hospitalarias disponibles por cada 1.000 habitantes en OPS una población, para un año dado, en un determinado país, territorio o área geográfica.

Número de médicos disponibles por cada 10.000 habitantes en una

OPS población, para un año dado, en un determinado país, territorio o área geográfica.

Razón de enfermeros

Número de enfermeros disponibles por cada 10.000 habitantes en una geográfica.

Razón de odontólogos

Número de odontólogos disponibles por cada 10.000 habitantes en una población, para un año dado, en un determinado país, territorio o área geográfica.

Fuentes: Elaboración propia con base en datos oficiales. OPS = Organización Panamericana de Salud; OMS = Organización Mundial de la Salud; SES = Sistema de Estadísticas de Salud (Argentina); CONEVAL= Consejo Nacional de Evaluación de la Política de Desarrollo Nacional (México).

Argentina: razón de consultas médicas, tasa de atención prenatal, razón de egresos hospitalarios, cantidad de establecimientos de salud por cada mil habitantes, razón de camas hospitalarias, razón de médicos, razón de enfermeros, razón de odontólogos, cobertura inmunizaciones DPT3, BCG y sarampión, tasa de atención profesional del parto, uso de anticonceptivos en mujeres, población cubierta por seguro de salud, porcentaje de 
Tabla 1. Continuación.

\begin{tabular}{|c|c|c|c|}
\hline \multirow{2}{*}{$\begin{array}{l}\text { Área } \\
\text { temática }\end{array}$} & \multicolumn{3}{|c|}{ Indicadores seleccionados } \\
\hline & Denominación & Definición & Institución \\
\hline \multirow[t]{8}{*}{$\begin{array}{l}\text { Acceso y } \\
\text { cobertura }\end{array}$} & Cobertura inmunizaciones DPT3 & $\begin{array}{l}\text { Proporción de niños de } 1 \text { año que recibieron tres dosis de vacuna contra la } \\
\text { difteria, el tétanos y la tos convulsiva. }\end{array}$ & OPS \\
\hline & Cobertura inmunizaciones BCG & Proporción de niños de 1 año vacunados contra la tuberculosis. & OPS \\
\hline & $\begin{array}{l}\text { Cobertura inmunizaciones } \\
\text { sarampión }\end{array}$ & Proporción de niños de 1 año vacunados contra el sarampión. & OPS \\
\hline & $\begin{array}{l}\text { Tasa de atención profesional del } \\
\text { parto }\end{array}$ & $\begin{array}{l}\text { Número de partos atendidos por personal capacitado durante un año } \\
\text { específico, independientemente de su lugar de ocurrencia, expresado como } \\
\text { porcentaje del número total de nacimientos en ese mismo año, en un } \\
\text { determinado país, territorio o área geográfica. }\end{array}$ & OPS \\
\hline & $\begin{array}{l}\text { Tiempo promedio de traslado al } \\
\text { hospital la última vez que se tuvo } \\
\text { una emergencia }\end{array}$ & $\begin{array}{l}\text { Promedio de tiempo de traslado a un establecimiento de salud, la última } \\
\text { vez que se tuvo una emergencia. }\end{array}$ & CONAVE \\
\hline & $\begin{array}{l}\text { Tiempo promedio estimado de } \\
\text { traslado al hospital en caso de } \\
\text { presentarse una emergencia }\end{array}$ & $\begin{array}{l}\text { Promedio de tiempo de traslado a un establecimiento de salud en caso de } \\
\text { emergencia. }\end{array}$ & CONAVE \\
\hline & Uso de anticonceptivos en mujeres & $\begin{array}{l}\text { Número de mujeres en edad fértil ( } 15 \text { a } 49 \text { años) que usan un método } \\
\text { anticonceptivo de cualquier tipo, en un punto de tiempo específico, } \\
\text { expresado como porcentaje de la correspondiente población a mitad de } \\
\text { año, para un año dado, en un determinado país, territorio o área geográfica. }\end{array}$ & OPS \\
\hline & $\begin{array}{l}\text { Población cubierta por seguro de } \\
\text { salud }\end{array}$ & $\begin{array}{l}\text { Población cubierta por obra social para un año dado, en un determinado } \\
\text { país, territorio o área geográfica. }\end{array}$ & OPS \\
\hline \multirow[t]{4}{*}{ Calidad } & $\begin{array}{l}\text { Porcentaje de personas que no } \\
\text { fueron atendidas al presentar un } \\
\text { problema de salud }\end{array}$ & $\begin{array}{l}\text { Porcentaje de personas que no fueron atendidas al presentar un problema } \\
\text { de salud. }\end{array}$ & CONAVE \\
\hline & $\begin{array}{l}\text { Motivo por el que las personas no } \\
\text { fueron atendidas al presentar un } \\
\text { problema de salud }\end{array}$ & $\begin{array}{l}\text { Porcentaje de población que no fue atendida al presentar un problema de } \\
\text { salud, según motivo de falta de atención. }\end{array}$ & CONAVE \\
\hline & $\begin{array}{l}\text { Tasa de infección de la herida } \\
\text { quirúrgica }\end{array}$ & $\begin{array}{l}\text { Tasa de infección de la herida quirúrgica, como porcentaje de todas las } \\
\text { operaciones quirúrgicas. }\end{array}$ & OMS \\
\hline & $\begin{array}{l}\text { Tasa de letalidad hospitalaria por } \\
\text { infarto agudo de miocardio a los } 30 \\
\text { días }\end{array}$ & $\begin{array}{l}\text { Proporción de pacientes hospitalizados con diagnóstico principal de infarto } \\
\text { agudo de miocardio (IAM) (accidente cerebrovascular) que murieron en un } \\
\text { plazo de } 30 \text { días después del ingreso. }\end{array}$ & OMS \\
\hline
\end{tabular}

Fuentes: Elaboración propia con base en datos oficiales. OPS = Organización Panamericana de Salud; OMS = Organización Mundial de la Salud; SES = Sistema de Estadísticas de Salud (Argentina); CONAVE= Consejo Nacional de Evaluación de la Política de Desarrollo Nacional (México).

personas que no fueron atendidas al presentar un problema de salud, motivo por el que las personas no fueron atendidas al presentar un problema de salud y tasa de infección de la herida quirúrgica.

Cabe resaltar que, de los indicadores mencionados, no es posible calcular la evolución a través del tiempo de solo uno (razón de odontólogos) porque el dato existe únicamente para el año 2004. A su vez, se destaca la interrupción de la serie en el caso de dos de ellos (razón de enfermeros y razón de odontólogos) al año 2004. Como contrapartida, resulta positivo el hecho de que pueda disponerse de al menos dos indicadores para cada área temática estudiada. 
Tabla 2. Disponibilidad de datos para calcular los indicadores seleccionados para monitoreo de servicios de salud en Argentina (total país), según área temática, disponibilidad y fuente. 2000-2016.

\begin{tabular}{|c|c|c|c|c|}
\hline \multirow{2}{*}{$\begin{array}{l}\text { Área } \\
\text { temática }\end{array}$} & \multirow{2}{*}{ Indicador } & \multicolumn{3}{|c|}{ Datos } \\
\hline & & Disponibilidad & Años disponibles & Fuentes \\
\hline \multirow[t]{7}{*}{ Utilización } & Razón de consultas médicas & Sí & $2000-2008,2010-2011$ & SES \\
\hline & Tasa de atención prenatal & Sí & $2001,2005,2012$ & SES \\
\hline & Razón de egresos hospitalarios & Sí & $2000-2010$ & SES \\
\hline & Porcentaje de ocupación & No & - & - \\
\hline & Giro de cama & No & - & - \\
\hline & Estancia promedio & No & - & - \\
\hline & $\begin{array}{l}\text { Atenciones por urgencia por } \\
\text { habitante }\end{array}$ & No & - & - \\
\hline \multirow[t]{5}{*}{ Disponibilidad } & $\begin{array}{l}\text { Cantidad de establecimientos de } \\
\text { salud por cada mil habitantes }\end{array}$ & Sí & 2000-2016 & SIISA \\
\hline & Razón de camas hospitalarias & Sí & $2000,2010-2013,2016$ & SIISA \\
\hline & Razón de médicos & Sí & $2004,2005,2010,2013$ & SES \\
\hline & Razón de enfermeros & Sí & 2001,2004 & SES \\
\hline & Razón de odontólogos & Sí & 2004 & SES \\
\hline \multirow{8}{*}{$\begin{array}{l}\text { Acceso y } \\
\text { cobertura }\end{array}$} & Cobertura inmunizaciones DPT3 & Sí & $2000-2013$ & SIISA \\
\hline & Cobertura inmunizaciones BCG & Sí & $2012-2013$ & SIISA \\
\hline & $\begin{array}{l}\text { Cobertura inmunizaciones } \\
\text { sarampión }\end{array}$ & Sí & $2000-2013$ & SIISA \\
\hline & $\begin{array}{l}\text { Tasa de atención profesional del } \\
\text { parto }\end{array}$ & Sí & $2000-2013$ & SES \\
\hline & $\begin{array}{l}\text { Tiempo promedio de traslado al } \\
\text { hospital la última vez que se tuvo } \\
\text { una emergencia }\end{array}$ & No & - & - \\
\hline & $\begin{array}{l}\text { Tiempo promedio estimado de } \\
\text { traslado al hospital en caso de } \\
\text { presentarse una emergencia }\end{array}$ & No & - & - \\
\hline & Uso de anticonceptivos en mujeres & Sí & $2001,2004,2005,2009$ & SES \\
\hline & $\begin{array}{l}\text { Población cubierta por seguro de } \\
\text { salud }\end{array}$ & Sí & 2001,2010 & $\begin{array}{c}\text { Censo } \\
\text { Nacional de } \\
\text { Población }\end{array}$ \\
\hline \multirow[t]{4}{*}{ Calidad } & $\begin{array}{l}\text { Porcentaje de personas que no } \\
\text { fueron atendidas al presentar un } \\
\text { problema de salud }\end{array}$ & Sí & 2005,2009 & ENFR \\
\hline & $\begin{array}{l}\text { Motivo por el que las personas no } \\
\text { fueron atendidas al presentar un } \\
\text { problema de salud }\end{array}$ & Sí & 2005,2009 & ENFR \\
\hline & $\begin{array}{l}\text { Tasa de infección de la herida } \\
\text { quirúrgica }\end{array}$ & Sí & 2004-2016 & SES \\
\hline & $\begin{array}{l}\text { Tasa de letalidad hospitalaria por } \\
\text { infarto agudo de miocardio a los } 30 \\
\text { días }\end{array}$ & No & - & - \\
\hline
\end{tabular}

Fuentes: Elaboración propia con base en datos oficiales. OPS = Organización Panamericana de Salud; SES = Sistema de Estadísticas de Salud (Argentina); ENFR = Encuesta Nacional de Factores de Riesgo (Argentina). 
La Tabla 2 permite inferir que, sobre todo a partir del año 2010, hubo un interés por mejorar la cantidad de datos en materia de salud pública, lo cual se refleja en la disponibilidad de fuentes. Sin embargo, los que más capacidad de elaboración presentan son los indicadores originados en sistemas internacionales, lo que invita a reflexionar acerca de si solo se está cumpliendo con los protocolos internacionales en vez de evidenciarse un real interés por monitorear y mejorar los servicios de salud del país.

Asimismo, es necesario evaluar la capacidad de desagregación de la información disponible para el cálculo de estos indicadores, empezando por el ámbito geográfico y temporal, pues ya ha sido evidenciado en otras investigaciones la existencia de amplias diferencias entre regiones, provincias y departamentos del territorio argentino a lo largo del tiempo ${ }^{(2,7)}$.

$\mathrm{Al}$ respecto, es bien sabido que los datos provenientes de fuentes internacionales, aun cuando son proporcionados por las autoridades de cada país, no incorporan desagregaciones según áreas menores u otras características, al menos en las publicaciones ofrecidas por dichas fuentes. Por el contrario, la información que puede obtenerse de las Direcciones Provinciales de Estadísticas en Salud a veces Ilega a ser abundante, aunque también menos homogénea en cuanto a cantidad y forma y más errática en cuanto a periodicidad.

En la Tabla 3 se aprecian las fuentes de información que presentan disponibilidad de datos para calcular los indicadores seleccionados a nivel provincial. Es necesario aclarar que aquellos indicadores que no están disponibles en todas las provincias, se consideran como no calculables.

De manera complementaria, la Tabla 4, Tabla 5, Tabla 6 y Tabla 7 exhiben la posibilidad de cálculo de los indicadores seleccionados según provincia y datos disponibles según año. Al analizar las tablas precedentes se observa que al tener en cuenta las estadísticas relevadas en el ámbito de la dirección nacional y provinciales de salud, pueden calcularse para todas las provincias solo 16 de los 24 indicadores seleccionados: razón de consultas médicas, tasa de atención prenatal, razón de egresos hospitalarios, cantidad de establecimientos de salud por cada mil habitantes, razón de camas hospitalarias, razón de médicos, razón de enfermeros, cobertura inmunizaciones DPT3, BCG y sarampión, tasa de atención profesional del parto, uso de anticonceptivos en mujeres, población cubierta por seguro de salud, porcentaje de personas que no fueron atendidas al presentar un problema de salud, motivo por el que las personas no fueron atendidas al presentar un problema de salud, y tasa de infección de la herida quirúrgica.

De estos indicadores, en algunos casos no es factible medir la evolución a través del tiempo por contar con datos para un solo año, a diferencia de lo que sucede con esos mismos indicadores para el total del país. Tales casos comprenden la tasa de atención prenatal (cuya evolución solo puede medirse en las provincias de Jujuy y San Luis), los indicadores de cobertura de inmunización (disponibles para un solo año, excepto en la Ciudad Autónoma de Buenos Aires, y las provincias de Buenos Aires, Corrientes, Chubut, Jujuy, Neuquén y San Juan) y la cantidad de médicos y enfermeros (que cuentan con más de un dato para las provincias de Corrientes, Jujuy, Misiones, Neuquén, Río Negro, Salta, San Juan, San Luis y Tierra del Fuego).

Existen cuatro indicadores que pueden calcularse solo para algunas provincias, pero no para el total del país, puesto que faltarían datos para lograr un promedio. Uno de ellos es la cantidad de atenciones por urgencia por habitante, que puede medirse en Jujuy, Misiones, Mendoza y Santa Fe (estas últimas dos provincias incluso pueden evaluar su evolución en el tiempo); y, los indicadores relacionados a la utilización de servicios de internación hospitalaria como son el porcentaje de ocupación de camas, el giro promedio de camas y la estancia promedio, que únicamente pueden obtenerse en la Ciudad Autónoma de Buenos Aires, y en las provincias de Buenos Aires, Córdoba, Corrientes, Chubut, Jujuy (solo para 2012), Mendoza, Misiones, Río Negro, Salta, San Luis, Santa Fe y Tierra del Fuego. 
Tabla 3. Disponibilidad de datos para calcular los indicadores seleccionados para monitoreo de servicios de salud en todas las provincias. Argentina, 2000-2016.

\begin{tabular}{|c|c|c|c|}
\hline \multirow{2}{*}{$\begin{array}{l}\text { Área } \\
\text { temática }\end{array}$} & \multirow{2}{*}{ Indicador } & \multicolumn{2}{|c|}{ Datos } \\
\hline & & Disponibilidad & Fuentes \\
\hline \multirow[t]{7}{*}{ Utilización } & Razón de consultas médicas & Sí & EUGSS \\
\hline & Tasa de atención prenatal & Sí & ENNyS \\
\hline & Razón de egresos hospitalarios & Sí & SES \\
\hline & Porcentaje de ocupación & No & - \\
\hline & Giro de cama & No & - \\
\hline & Estancia promedio & No & - \\
\hline & $\begin{array}{l}\text { Atenciones por urgencia por } \\
\text { habitante }\end{array}$ & No & - \\
\hline \multirow[t]{5}{*}{ Disponibilidad } & $\begin{array}{l}\text { Cantidad de establecimientos de } \\
\text { salud por cada mil habitantes }\end{array}$ & Sí & SES \\
\hline & Razón de camas hospitalarias & Sí & SES \\
\hline & Razón de médicos & Sí & SES \\
\hline & Razón de enfermeros & Sí & SES \\
\hline & Razón de odontólogos & No & - \\
\hline \multirow{8}{*}{$\begin{array}{l}\text { Acceso y } \\
\text { cobertura }\end{array}$} & Cobertura inmunizaciones DPT3 & Sí & SES \\
\hline & Cobertura inmunizaciones BCG & Sí & SES \\
\hline & $\begin{array}{l}\text { Cobertura inmunizaciones } \\
\text { sarampión }\end{array}$ & Sí & SES \\
\hline & $\begin{array}{l}\text { Tasa de atención profesional del } \\
\text { parto }\end{array}$ & Sí & SES \\
\hline & $\begin{array}{l}\text { Tiempo promedio de traslado al } \\
\text { hospital la última vez que se tuvo } \\
\text { una emergencia }\end{array}$ & No & - \\
\hline & $\begin{array}{l}\text { Tiempo promedio estimado de } \\
\text { traslado al hospital en caso de } \\
\text { presentarse una emergencia }\end{array}$ & No & - \\
\hline & Uso de anticonceptivos en mujeres & Sí & ENFR \\
\hline & $\begin{array}{l}\text { Población cubierta por seguro de } \\
\text { salud }\end{array}$ & Sí & $\begin{array}{l}\text { Censo Nacional de } \\
\text { Población }\end{array}$ \\
\hline \multirow[t]{4}{*}{ Calidad } & $\begin{array}{l}\text { Porcentaje de personas que no } \\
\text { fueron atendidas al presentar un } \\
\text { problema de salud }\end{array}$ & Sí & ENFR \\
\hline & $\begin{array}{l}\text { Motivo por el que las personas no } \\
\text { fueron atendidas al presentar un } \\
\text { problema de salud }\end{array}$ & Sí & ENFR \\
\hline & $\begin{array}{l}\text { Tasa de infección de la herida } \\
\text { quirúrgica }\end{array}$ & Sí & SES \\
\hline & $\begin{array}{l}\text { Tasa de letalidad hospitalaria por } \\
\text { infarto agudo de miocardio a los } 30 \\
\text { días }\end{array}$ & No & - \\
\hline
\end{tabular}

Fuentes: Elaboración propia con base en datos oficiales. EUGSS = Encuesta de Utilización y Gastos en Servicios de Salud (Argentina); ENNyS = Encuesta Nacional de Nutrición y Salud (Argentina); SES = Sistema de Estadísticas de Salud (Argentina); ENFR = Encuesta Nacional de Factores de Riesgo (Argentina). 
Tabla 4. Disponibilidad de datos para calcular indicadores seleccionados de utilización para monitoreo de servicios de salud, por jurisdicción y años. Argentina, 2000-2015.

\begin{tabular}{|c|c|c|c|c|c|c|c|}
\hline \multirow[b]{2}{*}{ JURISDICCIONES } & \multicolumn{7}{|c|}{ INDICADORES DE UTILIZACIÓN } \\
\hline & $\begin{array}{l}\text { Razón de } \\
\text { consultas } \\
\text { médicas }\end{array}$ & $\begin{array}{l}\text { Tasa de } \\
\text { atención } \\
\text { prenatal }\end{array}$ & $\begin{array}{c}\text { Razón de } \\
\text { egresos } \\
\text { hospitalarios }\end{array}$ & $\begin{array}{c}\text { Porcentaje } \\
\text { de ocupación }\end{array}$ & $\begin{array}{l}\text { Giro de } \\
\text { cama }\end{array}$ & $\begin{array}{l}\text { Estancia } \\
\text { promedio }\end{array}$ & $\begin{array}{c}\text { Atenciones por } \\
\text { urgencia por } \\
\text { habitante }\end{array}$ \\
\hline CABA & $2000-2013$ & 2004-2005 & $2000-2013$ & $2000-2013$ & $2000-2013$ & $2000-2013$ & - \\
\hline Buenos Aires & $\begin{array}{l}2000-2004 \\
2008-2012\end{array}$ & 2004-2005 & $2000-2013$ & $2008-2012$ & $2008-2012$ & $2008-2012$ & - \\
\hline Catamarca & $2000-2004$ & 2004-2005 & $2000-2010$ & - & - & - & - \\
\hline \multirow{2}{*}{ Córdoba } & $2000-2004$ & \multirow{2}{*}{ 2004-2005 } & $2000-2010$ & \multirow{2}{*}{$2007-2013$} & \multirow{2}{*}{$2007-2013$} & \multirow{2}{*}{ 2007-2013 } & \multirow{2}{*}{-} \\
\hline & $2007-2013$ & & $2007-2013$ & & & & \\
\hline \multirow{2}{*}{ Corrientes } & $2000-2012$ & \multirow{2}{*}{ 2004-2005 } & $2000-2012$ & $2000-2012$ & $2000-2012$ & $2000-2012$ & \multirow{2}{*}{$\begin{array}{l}- \\
-\end{array}$} \\
\hline & $2000-2012$ & & $2000-2012$ & $2000-2012$ & $2000-2012$ & $2000-2012$ & \\
\hline Chaco & $2000-2004$ & 2004-2005 & $2000-2010$ & - & - & - & - \\
\hline \multirow{2}{*}{ Chubut } & \multirow{2}{*}{$2000-2008$} & \multirow{2}{*}{ 2004-2005 } & $2000-2010$ & \multirow{2}{*}{$2005-2013$} & \multirow{2}{*}{$2005-2013$} & \multirow{2}{*}{$2005-2013$} & \multirow{2}{*}{ - } \\
\hline & & & $2000-2008$ & & & & \\
\hline Entre Ríos & $2000-2004$ & 2004-2005 & $2000-2010$ & - & - & - & - \\
\hline Formosa & $2000-2004$ & 2004-2005 & $2000-2010$ & - & - & - & - \\
\hline \multirow{2}{*}{ Jujuy } & $2000-2004$ & $2004-2005$ & $2000-2010$ & \multirow{2}{*}{2012} & \multirow{2}{*}{2012} & \multirow{2}{*}{2012} & \multirow{2}{*}{2012} \\
\hline & 2012 & 2012 & 2012 & & & & \\
\hline La Pampa & $2000-2004$ & 2004-2005 & $2000-2010$ & - & - & - & - \\
\hline La Rioja & $2000-2004$ & 2004-2005 & $2000-2010$ & - & - & - & - \\
\hline Mendoza & $2000-2013$ & 2004-2005 & $2000-2013$ & $2000-2013$ & $2000-2013$ & $2000-2013$ & $2000-2013$ \\
\hline Misiones & $2000-2004$ & 2004-2005 & $2000-2010$ & $2009-2012$ & 2009-2012 & 2009-2012 & 2012 \\
\hline Neuquén & $2000-2012$ & 2004-2005 & $2000-2010$ & - & - & - & - \\
\hline \multirow{2}{*}{ Río Negro } & \multirow{2}{*}{$2000-2004$} & \multirow{2}{*}{ 2004-2005 } & $2000-2010$ & \multirow{2}{*}{$2003-2013$} & \multirow{2}{*}{$2003-2013$} & $2002-2012$ & 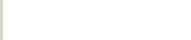 \\
\hline & & & $2003-2013$ & & & $2000-2015$ & 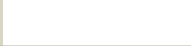 \\
\hline Salta & $2000-2011$ & 2004-2005 & $2000-2011$ & $2000-2011$ & $2000-2011$ & $2000-2011$ & - \\
\hline San Juan & $2000-2007$ & 2004-2005 & $2000-2010$ & - & - & - & - \\
\hline Son Luic t & $2000-2004$ & 2004-2005 & $2000-2010$ & $2012-2014$ & $2012-2014$ & $2012-2014$ & 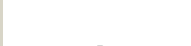 \\
\hline 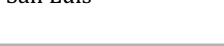 & $2012-2014$ & $2012-2014$ & $2012-2014$ & $2012-2011$ & 2014 2014 & 2010 & \\
\hline Santa Cruz & $2000-2004$ & 2004-2005 & $2000-2010$ & - & - & - & - \\
\hline & & & $2000-2010$ & & & & \\
\hline santa re & $2000-2009$ & $2004-\angle 005$ & $2000-2009$ & $2005-2009$ & $2005-2009$ & $2005-\angle 009$ & $2005-2009$ \\
\hline Santiago del Estero & $2000-2004$ & 2004-2005 & $2000-2010$ & - & - & - & - \\
\hline Tucumón & $2000-2004$ & $2004-2005$ & $2000-2010$ & & & & \\
\hline 1 uevinanit & $2000-2004$ & $2004-2000$ & 2004-2011 & - & - & - & 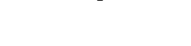 \\
\hline Tierre del Fueq & 2000-2004, & $2004-2005$ & $2000-2010$ & 2010-2012 & $2010-2012$ & 2010-2012 & 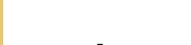 \\
\hline 11ент der ruego & 2012 & $2004-2005$ & $2010-2012$ & $2010-2012$ & $2010-2012$ & $2010-2012$ & - \\
\hline
\end{tabular}


Tabla 5. Disponibilidad de datos para calcular indicadores seleccionados de disponibilidad para monitoreo de servicios de salud, por jurisdicción y años. Argentina, 2000-2015.

\begin{tabular}{|c|c|c|c|c|c|}
\hline \multirow[b]{2}{*}{ JURISDICCIONES } & \multicolumn{5}{|c|}{ INDICADORES DE DISPONIBILIDAD } \\
\hline & $\begin{array}{c}\text { Cantidad de } \\
\text { establecimientos de } \\
\text { salud }\end{array}$ & $\begin{array}{l}\text { Razón de camas } \\
\text { hospitalarias }\end{array}$ & Razón de médicos & $\begin{array}{l}\text { Razón de } \\
\text { enfermeros }\end{array}$ & $\begin{array}{c}\text { Razón de } \\
\text { odontólogos }\end{array}$ \\
\hline \multirow{2}{*}{ CABA } & $2000,2008-2012$ & \multirow{2}{*}{$\begin{array}{c}2000,2003-2013 \\
2015\end{array}$} & \multirow{2}{*}{2001} & \multirow{2}{*}{2001} & \multirow{2}{*}{ - } \\
\hline & 2015 & & & & \\
\hline \multirow{2}{*}{ Buenos Aires } & 2000 & \multirow{2}{*}{$\begin{array}{c}2000,2008-2012 \\
2015\end{array}$} & \multirow{2}{*}{2001} & \multirow{2}{*}{2001} & \multirow{2}{*}{ - } \\
\hline & 2015 & & & & \\
\hline \multirow{2}{*}{ Catamarca } & $2000,2007-2013$ & \multirow{2}{*}{2000,2015} & \multirow{2}{*}{2001} & \multirow{2}{*}{2001} & \multirow{2}{*}{-} \\
\hline & 2015 & & & & \\
\hline \multirow{2}{*}{ Córdoba } & $2000,2003-2012$ & 2000,2015 & \multirow{2}{*}{2001} & \multirow{2}{*}{2001} & \multirow{2}{*}{ - } \\
\hline & 2015 & $2007-2013$ & & & \\
\hline \multirow{3}{*}{ Corrientes } & \multirow{2}{*}{2000} & $2000-2012$ & \multirow{3}{*}{$2001,2006-2009$} & \multirow{3}{*}{$2001,2006-2009$} & \multirow{3}{*}{$2006-2009$} \\
\hline & & $2000-2012$ & & & \\
\hline & 2015 & 2001-2010 & & & \\
\hline \multirow{3}{*}{ Chaco } & 2000 & \multirow{3}{*}{2000,2015} & \multirow{3}{*}{2001} & \multirow{3}{*}{2001} & \multirow{3}{*}{ - } \\
\hline & $2005-2013$ & & & & \\
\hline & 2015 & & & & \\
\hline & 2000 & $2000-2013$ & & & \\
\hline Chubut & 2010 & 0015 & 2001 & 2001 & - \\
\hline & 2015 & & & & \\
\hline Гntro D'o & 2000 & 2000,2015 & 2001 & 2001 & \\
\hline Litlic TIUS & 2015 & 2010 & & & \\
\hline 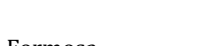 & 2000,2012 & r & 2001 & 2001 & \\
\hline rolitiosd & 2015 & 2000,2010 & 2001 & 2001 & \\
\hline & 2000 & 20002015 & 2001 & 2001 & \\
\hline Jujuy & 2013 & 2000,2015 & 2001 & 2001 & \\
\hline & 2015 & 2012 & 2012 & 2012 & 2012 \\
\hline & (2) & 2000,2015 & & & \\
\hline La Pampa & 2000 & 2013 & 2001 & 2001 & - \\
\hline & 2015 & 2013 & & & \\
\hline & 2000 & & & & \\
\hline La Kioja & 2015 & 2000,2015 & 2001 & 2001 & - \\
\hline
\end{tabular}


Tabla 5. Continuación.

\begin{tabular}{|c|c|c|c|c|c|}
\hline \multirow{2}{*}{ JURISDICCIONES } & \multicolumn{5}{|c|}{ INDICADORES DE DISPONIBILIDAD } \\
\hline & $\begin{array}{l}\text { Cantidad de } \\
\text { establecimientos de } \\
\text { salud }\end{array}$ & $\begin{array}{l}\text { Razón de camas } \\
\text { hospitalarias }\end{array}$ & Razón de médicos & $\begin{array}{l}\text { Razón de } \\
\text { enfermeros }\end{array}$ & $\begin{array}{c}\text { Razón de } \\
\text { odontólogos }\end{array}$ \\
\hline \multirow{2}{*}{ Mendoza } & 2000,2011 & 2015 & \multirow{2}{*}{2001} & \multirow{2}{*}{2001} & \multirow{2}{*}{ - } \\
\hline & 2015 & 2001-2013 & & & \\
\hline \multirow{2}{*}{ Misiones } & $1995-2012$ & 2000,2015 & \multirow{2}{*}{2001,2011} & \multirow{2}{*}{2001,2011} & \multirow{2}{*}{2001} \\
\hline & 2015 & 2009-2012 & & & \\
\hline \multirow{3}{*}{ Neuquén } & 2000 & \multirow{2}{*}{2015} & & \multirow{3}{*}{$2000-2010$} & \multirow{3}{*}{$2000-2010$} \\
\hline & $2003-2013$ & & $2000-2010$ & & \\
\hline & 2015 & $2002-2012$ & & & \\
\hline \multirow{2}{*}{ Río Negro } & 2000,2011 & 2000,2015 & 2001 & 2001 & \multirow{2}{*}{$2003-2013$} \\
\hline & 2015 & $2003-2013$ & $2003-2013$ & $2003-2013$ & \\
\hline \multirow{2}{*}{ Salta } & 2000,2013 & \multirow{2}{*}{2000 a 2011,2015} & \multirow{2}{*}{2000 a 2010} & \multirow{2}{*}{2000 a 2010} & \multirow{2}{*}{2000 a 2010} \\
\hline & 2015 & & & & \\
\hline \multirow{3}{*}{ San Juan } & 2000 & \multirow{3}{*}{$2000,2013,2015$} & \multirow{3}{*}{2001 y 2013} & \multirow{3}{*}{2001 y 2013} & \multirow{3}{*}{2013} \\
\hline & $2012-2014$ & & & & \\
\hline & 2015 & & & & \\
\hline \multirow{2}{*}{ San Luis } & 2000 & 2000,2015 & 2001 & 2001 & - \\
\hline & 2015 & $2012-2014$ & $2012-2014$ & $2012-2014$ & $2012-2014$ \\
\hline \multirow{3}{*}{ Santa Cruz } & 2000 & \multirow{3}{*}{2000,2015} & \multirow{3}{*}{2001} & \multirow{3}{*}{2001} & \multirow{3}{*}{-} \\
\hline & $2005-2009$ & & & & \\
\hline & 2015 & & & & \\
\hline Canto Eo & 2000 & 2000,2015 & 2001 & 2001 & \\
\hline Santa re & 2015 & $2005-2009$ & 2001 & 2001 & - \\
\hline Sontiou dol Fetoro & 2000 & 20002015 & 2001 & 2001 & \\
\hline Santiago uer Lstero & 2015 & 2000,2013 & 2001 & 2001 & \\
\hline T. & 2000 & 20002015 & 2001 & 2001 & \\
\hline I ucumañ & 2015 & 2000,2015 & 2001 & 2001 & \\
\hline$\pi \cdot$ & 2000 & 2000,2015 & & & \\
\hline 1IEIId uer ruego & 2015 & 2010-2012 & 2000,2001 & 2000,2001 & 2000 \\
\hline
\end{tabular}

Fuentes: Elaboración propia con base en datos de la Dirección de Estadísticas e Información de Salud (DEIS), Sistema Estadístico de Salud (SES), Ministerio de Salud de la Nación. 
Tabla 6. Disponibilidad de datos para calcular indicadores seleccionados de acceso y cobertura para monitoreo de servicios de salud, por jurisdicción y años. Argentina, 2000-2015.

\begin{tabular}{|c|c|c|c|c|c|c|}
\hline \multirow[b]{2}{*}{ JURISDICCIONES } & \multicolumn{6}{|c|}{ INDICADORES DE ACCESO Y COBERTURA } \\
\hline & $\begin{array}{c}\text { Cobertura } \\
\text { inmunizaciones } \\
\text { DPT3 }\end{array}$ & $\begin{array}{c}\text { Cobertura } \\
\text { inmunizaciones } \\
\text { BCG }\end{array}$ & $\begin{array}{c}\text { Cobertura } \\
\text { inmunizaciones } \\
\text { sarampión }\end{array}$ & $\begin{array}{c}\text { Tasa de atención } \\
\text { profesional del } \\
\text { parto }\end{array}$ & $\begin{array}{c}\text { Uso de } \\
\text { anticonceptivos } \\
\text { en mujeres }\end{array}$ & $\begin{array}{c}\text { Población } \\
\text { cubierta por } \\
\text { seguro de salud }\end{array}$ \\
\hline CABA & $2005-2013$ & $2005-2013$ & $2005-2013$ & $2000-2004$ & $2005-2009$ & 2001,2010 \\
\hline Buenos Aires & $2008-2013$ & $2008-2013$ & 2008-2013 & $2000-2004$ & 2005-2009 & 2001,2010 \\
\hline Catamarca & 2013 & 2013 & 2013 & $2000-2004$ & $2005-2009$ & 2001,2010 \\
\hline Córdoba & 2013 & 2013 & 2013 & $2000-2004$ & $2005-2009$ & 2001,2010 \\
\hline \multirow{3}{*}{ Corrientes } & & & & $2000-2004$ & \multirow{3}{*}{$2005-2009$} & \multirow{3}{*}{2001,2010} \\
\hline & 2013 & 2013 & 2013 & $2003-2012$ & & \\
\hline & 2003 & 2003 & 2003 & $2003-2012$ & & \\
\hline Chaco & 2013 & 2013 & 2013 & $2000-2004$ & $2005-2009$ & 2001,2010 \\
\hline Chubut & $2005-2013$ & $2005-2013$ & $2005-2013$ & $2000-2008$ & $2005-2009$ & 2001,2010 \\
\hline Entre Ríos & 2013 & 2013 & 2013 & $2000-2004$ & $2005-2009$ & 2001,2010 \\
\hline Formosa & 2013 & 2013 & 2013 & $2000-2004$ & 2005-2009 & 2001,2010 \\
\hline \multirow{2}{*}{ Jujuy } & 2013 & 2013 & 2013 & $2000-2004$ & \multirow{2}{*}{$2005-2009$} & \multirow{2}{*}{2001,2010} \\
\hline & 2012 & 2012 & 2012 & 2012 & & \\
\hline La Pampa & 2013 & 2013 & 2013 & $2000-2004$ & $2005-2009$ & 2001,2010 \\
\hline La Rioja & 2013 & 2013 & 2013 & $2000-2004$ & $2005-2009$ & 2001,2010 \\
\hline Mendoza & 2013 & 2013 & 2013 & $2000-2004$ & $2005-2009$ & 2001,2010 \\
\hline Misiones & 2013 & 2013 & 2013 & $2000-2004$ & 2005-2009 & 2001,2010 \\
\hline Neuquén & $1992-2013$ & $1992-2013$ & 1992-2013 & $2000-2012$ & 2005-2009 & 2001,2010 \\
\hline Río Negro & 2013 & 2013 & 2013 & $2000-2004$ & $2005-2009$ & 2001,2010 \\
\hline Salta & 2013 & 2013 & 2013 & $2000-2004$ & $2005-2009$ & 2001,2010 \\
\hline San Juan & $2009-2013$ & 2009-2013 & $2009-2013$ & $2000-2004$ & $2005-2009$ & 2001,2010 \\
\hline San Luis & 2013 & 2013 & 2013 & $2000-2004$ & $2005-2009$ & 2001,2010 \\
\hline Santa Cruz & 2013 & 2013 & 2013 & $2000-2004$ & $2005-2009$ & 2001,2010 \\
\hline Santa Fe & 2013 & 2013 & 2013 & $2000-2004$ & 2005-2009 & 2001,2010 \\
\hline Santiago del Estero & 2013 & 2013 & 2013 & $2000-2004$ & $2005-2009$ & 2001,2010 \\
\hline Tucumán & 2013 & 2013 & 2013 & $2000-2004$ & $2005-2009$ & 2001,2010 \\
\hline Tierra del Fuego & 2013 & 2013 & 2013 & $2000-2004$ & $2005-2009$ & 2001,2010 \\
\hline
\end{tabular}


Tabla 7. Disponibilidad de datos para calcular indicadores seleccionados de calidad para monitoreo de servicios de salud, por jurisdicción y años. Argentina 2000-2015.

\begin{tabular}{|c|c|c|}
\hline \multirow{2}{*}{ JURISDICCIONES } & \multicolumn{2}{|c|}{ INDICADORES DE CALIDAD } \\
\hline & $\begin{array}{l}\text { Porcentaje de personas que no } \\
\text { fueron atendidas al presentar un } \\
\text { problema de salud }\end{array}$ & $\begin{array}{l}\text { Motivo por el que las personas no } \\
\text { fueron atendidas al presentar un } \\
\text { problema de salud }\end{array}$ \\
\hline CABA & 2005,2009 & 2005,2009 \\
\hline Buenos Aires & 2005,2009 & 2005,2009 \\
\hline Catamarca & 2005,2009 & 2005,2009 \\
\hline Córdoba & 2005,2009 & 2005,2009 \\
\hline Corrientes & 2005,2009 & 2005,2009 \\
\hline Chaco & 2005,2009 & 2005,2009 \\
\hline Chubut & 2005,2009 & 2005,2009 \\
\hline Entre Ríos & 2005,2009 & 2005,2009 \\
\hline Formosa & 2005,2009 & 2005,2009 \\
\hline Jujuy & 2005,2009 & 2005,2009 \\
\hline La Pampa & 2005,2009 & 2005,2009 \\
\hline La Rioja & 2005,2009 & 2005,2009 \\
\hline Mendoza & 2005,2009 & 2005,2009 \\
\hline Misiones & 2005,2009 & 2005,2009 \\
\hline Neuquén & 2005,2009 & 2005,2009 \\
\hline Río Negro & 2005,2009 & 2005,2009 \\
\hline Salta & 2005,2009 & 2005, 2009 \\
\hline San Juan & 2005,2009 & 2005,2009 \\
\hline San Luis & 2005,2009 & 2005,2009 \\
\hline Santa Cruz & 2005,2009 & 2005,2009 \\
\hline Santa Fe & 2005,2009 & 2005,2009 \\
\hline Santiago del Estero & 2005,2009 & 2005,2009 \\
\hline Tucumán & 2005,2009 & 2005,2009 \\
\hline Tierra del Fuego & 2005,2009 & 2005,2009 \\
\hline
\end{tabular}

Fuentes: Elaboración propia con base en datos de la Dirección de Estadísticas e Información de Salud (DEIS), Sistema Estadístico de Salud (SES), Ministerio de Salud de la Nación.

Por otro lado, existe un indicador que puede calcularse para el total del país, pero no para cada provincia, que es la razón de odontólogos. Este dato está disponible para Corrientes, Jujuy, Misiones, Neuquén, Río Negro, Salta, San Juan, San Luis y Tierra del Fuego. Pero mientras que a nivel país, para este indicador solo existen datos para el año 2004, a nivel provincial podemos observar su tendencia en Corrientes, Neuquén, Río Negro, Salta y San Luis.

En el caso de la tasa de infección de herida quirúrgica, una observación a ser tenida en cuenta es que si bien este indicador puede ser 
Tabla 8. Características de las fuentes de datos utilizadas para calcular los indicadores seleccionados para el monitoreo de los servicios de salud. Argentina, 2000-2015.

\begin{tabular}{|c|c|c|c|c|c|}
\hline \multirow{2}{*}{$\begin{array}{l}\text { CARACTERÍSTICAS } \\
\text { DE LAS FUENTES }\end{array}$} & \multicolumn{5}{|c|}{ FUENTES DE DATOS } \\
\hline & SES & ENNyS & ENFR & $\begin{array}{l}\text { Censos de } \\
\text { población }\end{array}$ & EUGSS \\
\hline Universo & Toda la población & Muestra & Muestra & Toda la población & Muestra \\
\hline Periodicidad & Continuo & $\begin{array}{l}\text { Edición única } \\
\text { (2004-2005) }\end{array}$ & 2005,2009 & Cada 10 años & $2003,2005,2010$ \\
\hline Cobertura & Todo el territorio & $\begin{array}{c}\text { Localidades de } \\
5.000 \text { y más } \\
\text { habitantes }\end{array}$ & - & Todo el territorio & - \\
\hline \multicolumn{6}{|l|}{ Desagregaciones admitidas } \\
\hline Geográfica & $\mathrm{X}$ & $\mathrm{X}$ & $\mathrm{x}$ & $\mathrm{X}$ & $\mathrm{x}$ \\
\hline Causas & $\mathrm{X}$ & - & - & - & $\mathrm{X}$ \\
\hline Edad & $\mathrm{X}$ & $\mathrm{X}$ & $\mathrm{x}$ & $\mathrm{X}$ & $\mathrm{X}$ \\
\hline Sexo & $\mathrm{X}$ & $\mathrm{X}$ & $\mathrm{X}$ & $\mathrm{X}$ & $\mathrm{X}$ \\
\hline Dependencia administrativa & $\mathrm{X}$ & - & - & - & $\mathrm{X}$ \\
\hline Diagnóstico & $\mathrm{X}$ & - & - & - & - \\
\hline Nivel Educativo & - & $\mathrm{X}$ & $\mathrm{x}$ & $\mathrm{X}$ & $\mathrm{X}$ \\
\hline Nivel de ingreso & - & $\mathrm{X}$ & - & - & $X$ \\
\hline
\end{tabular}

Fuentes: Elaboración propia con base en datos oficiales. SES = Sistema de Estadísticas de Salud (Argentina); ENNyS = Encuesta Nacional de Nutrición y Salud (Argentina); ENFR = Encuesta Nacional de Factores de Riesgo (Argentina); EUGSS = Encuesta de Utilización y Gastos en Servicios de Salud (Argentina).

calculado para todas las provincias y a través del tiempo, debemos mencionar que esta información no ha sido publicada ni es accesible para toda la población, sino con acceso restringido para los profesionales de la salud, los hospitales adheridos al Programa Nacional de Epidemiología y Control de Infecciones Hospitalarias (VIHDA) de Argentina, las autoridades nacionales y jurisdiccionales y al personal del Instituto Nacional de Epidemiología “Dr. Juan H. Jara".

A pesar de este obstáculo, es importante destacar la importancia que reviste la conformación de redes de investigación impulsadas por sociedades civiles, en coordinación con los Ministerios de Salud y otras instituciones del sistema, en torno a los sistemas de información en salud, a fin de fortalecer el liderazgo en la producción y uso de la información ${ }^{(6)}$.

Teniendo en cuenta la capacidad de desagregación de los territorios provinciales en departamentos, localidades, áreas sanitarias o programáticas, u hospitales, son pocas las provincias que ofrecen este tipo de disponibilidad siendo, en su mayoría, datos para años más recientes, y sin homogeneidad en cuanto a este nivel de desagregación. En 
efecto, solo encontramos cinco provincias que poseen información desagregada geográficamente desde casi principios de siglo: Corrientes, Chubut, Mendoza, Neuquén y Santa Fe. Otras tres provincias (Córdoba, San Luis y Tierra del Fuego) poseen esa desagregación para los últimos 4 ó 5 años del período estudiado. En el caso de la provincia de Jujuy, posee información desagregada solo para el año 2012.

A su vez, gran parte de los indicadores, sobre todo los de calidad y algunos de los de acceso y cobertura, dependen de encuestas y censos realizados no siempre con la misma periodicidad. Esta situación se agrava al considerar que el SES no publica ni brinda acceso público a la desagregación de muchos de sus indicadores.

Más allá de lo expuesto, puede observarse en los datos provinciales la misma tendencia que se da a nivel de país, esto es, un aumento en la disponibilidad de los datos para los últimos años.

Siguiendo con los objetivos planteados al principio de este artículo, y habiendo sido ya mencionado el tema de la desagregación geográfica y temporal, resta evaluar otras variables de desagregación de los datos, así como las características de publicación de los mismos. Esto se realiza en la Tabla 8, donde se exhiben algunas características de las fuentes de datos utilizadas.

Es necesario aclarar que, en el caso del SES, la desagregación que se muestra no está disponible para los datos de cobertura de inmunizaciones y la tasa de atención prenatal, así como tampoco para indicadores que, por su naturaleza, no permiten ciertas desagregaciones como, por ejemplo, la cantidad de establecimientos de salud por 1.000 habitantes o la razón de médicos por habitante (que no admiten desagregaciones por sexo o edad). Sin embargo, es una de las pocas fuentes de datos capaces de recolectar registros sobre mortalidad y morbilidad.

Con respecto a las otras fuentes, se trata de encuestas específicas a población (excepto los censos), que no cuentan con una periodicidad definida (como en el caso de la ENNyS) y sus datos se corresponden a una muestra poblacional, por lo que existen limitaciones en su generalización a todo el territorio.

Finalmente, los censos constituyen una fuente inmejorable en cuanto a representatividad poblacional y desagregación de todos sus datos. Sin embargo, la cantidad de información que puede obtenerse en materia de salud es ínfima, y presentan una periodicidad decenal, cuestión que impide la toma de decisiones de corto plazo y la evaluación de los respectivos resultados. Resulta prudente mencionar que todas estas fuentes son accesibles a la población en general.

\section{DISCUSIÓN}

El fortalecimiento de los sistemas de salud constituye una de las cuatro orientaciones estratégicas de las OMS, expuestas en el Informe sobre la salud en el mundo 2000 y reafirmada en el informe de 2003. Este último documento afirma, además, que la insuficiencia de la información sanitaria es uno de los grandes retos afrontados por los sistemas de salud para promover la equidad ${ }^{(6)}$.

A los fines de suplir la falta de información en salud, y ante la necesidad de coordinar y alinear asociaciones para el desarrollo de los sistemas de salud, la OMS lanzó en 2005 las Health Metrics Network (HMN). Estas redes en métricas de salud proponían el establecimiento de indicadores en el sistema de información en salud que brinden información oportuna para dar seguimiento a los avances y establecer cambios en los servicios y políticas de salud ${ }^{(8)}$ :

...cada país necesitará un paquete mínimo de indicadores de salud que serán útiles en la planificación, monitoreo y evaluación. A pesar de que los indicadores son necesarios para monitorear las prioridades nacionales, estos también deben cumplir con los estándares internacionales. Los indicadores nacionales deben estar vinculados y armonizados con los indicadores de las iniciativas internacionales y globales, tales como los Objetivos 
de Desarrollo del Milenio. [Traducción del original: All countries therefore need a nationally defined minimum set of health indicators used regularly in national programme planning, monitoring and evaluation. Although health indicators are needed to monitor local and national priorities, indicator definitions must also meet international technical standards. Moreover, national indicators should be consistently linked and harmonized with key indicators in major international and global initiatives, such as the MDGs]

Entre estos indicadores de salud se incluyen aquellos relacionados con los servicios de salud, que son el objetivo de esta investigación. Se supone que los mecanismos estatales e institucionales deben garantizar el acceso a los servicios de salud y la prestación y financiación de esta, sobre todo ante las situaciones de crisis económicas y/o políticas. Entonces, la disponibilidad de información periódica y desagregada referida a los servicios de salud de un territorio o población constituye una necesidad para su gerenciamiento y la distribución de los recursos financieros para tal fin; además, determina oportunidades de mejoras de estos servicios.

En muchos casos, esta información es generada a través de diferentes sistemas y fuentes de información que no son accesibles de manera tal que puedan ser fácilmente recolectadas y comparadas ${ }^{(6)}$.

En Argentina, no hay trabajos anteriores que indaguen sobre el monitoreo de indicadores de servicios de salud, aunque sí existen otras experiencias de evaluación que pueden ser utilizadas como antecedentes del tema, por ejemplo la llevada a cabo por Camilo Marracino et al., en el año 2000, que analiza exclusivamente los indicadores para un monitoreo de planes de cobertura ${ }^{(9)}$. Más recientemente, Eleonora Rojas Cabrera ${ }^{(2)}$ investiga las posibilidades del sistema de información oficial en Argentina para el monitoreo del ejercicio del derecho a la salud en la primera infancia, desde la perspectiva del acceso a las prestaciones y servicios comprometidos por el Estado en el ámbito internacional. Este último trabajo se postula como antecedente directo de la actual investigación, pues evalúa la capacidad del sistema estadístico oficial argentino de obtención de indicadores de salud, en el marco de los derechos humanos.

En Latinoamérica, existen diversas experiencias, como el estudio llevado a cabo en México por el Consejo Nacional de Evaluación de la Política de Desarrollo Social (CONEVAL) en 2012, en el que presenta una propuesta de indicadores de acceso y uso efectivo de los servicios de salud, con base en fuentes de datos públicas, para el seguimiento y evaluación del Sistema de Protección Social en Salud ${ }^{(10)}$. El Fondo Nacional de Salud (FONASA) de Chile también ha realizado, en los últimos años, un esfuerzo sistemático para clarificar las transferencias a los servicios de salud, considerando que es fundamental analizar los indicadores de gestión que permitan un uso más eficiente de los recursos asignados a estos servicios ${ }^{(11)}$.

Con base en la escasa bibliografía sobre la temática, surge el interrogante de si en Argentina se está poniendo poco énfasis en la recolección de datos sobre indicadores de acceso y uso a los servicios de salud en comparación con otros indicadores, como los que evalúan el estado de salud de la población (mortalidad infantil, esperanza de vida, y otros). Una hipótesis que se plantea es que esta falencia en la adquisición de datos podría deberse a que se priorizan los cambios en los indicadores del estado de salud de la población para estimar, indirectamente, la mejora en el sistema de salud. Los cambios en tales indicadores se deben a la aplicación efectiva de programas específicos de salud, que no generan en realidad reformas concretas en el sistema de salud. Si tal fuera el caso, evidenciaría un desinterés por el mejoramiento de los sistemas de salud, tanto en su organización como en su financiamiento.

En consecuencia, a los fines de afrontar los continuos retos en salud pública y, a su vez, adoptar medidas que permitan mejorar significativamente los resultados de las intervenciones que se realizan, los países deben determinar cuáles son las lagunas de información de las que adolecen y abordarlas, tanto en los sectores públicos como privados ${ }^{(12)}$. 


\section{CONCLUSIONES}

Según el análisis realizado, la información disponible a nivel oficial en el país no permite calcular la totalidad de indicadores, lo que evidencia la necesidad de incorporar nuevas fuentes, o bien adaptar las existentes. En algunos casos, los indicadores solo pueden ser construidos para un momento en el tiempo, lo que impide el monitoreo de avances, la detección de desafíos y desafíos y, simultáneamente, la evaluación de resultados de programas dirigidos a ese ámbito. Por otro lado, la falta de información desagregada dificulta indagar la situación de determinados grupos poblacionales, en lo concerniente a acceso a los servicios de salud.

Adicionalmente, casi toda la información acerca de los sistemas de salud se refiere solamente a la prestación de servicios o a la inversión en la atención sanitaria, los cuales generan gran parte del empleo y del gasto en salud. De acuerdo a la OMS, existen actividades que deberían incluirse en un concepto más amplio de lo que es un sistema de salud, las que tendrían que cuantificarse y evaluarse a fin de analizar la forma en que impactan sobre la salud de las personas ${ }^{(13)}$.

\section{REFERENCIAS BIBLIOGRÁFICAS}

1. Rodriguez Acosta S. Barreras y determinantes del acceso a los servicios de salud en Colombia [Tesis de Maestría]. Barcelona: Programa de Máster de Investigación en Economía Aplicada, Departament d'Economia Aplicada, Universitat Autònoma de Barcelona; 2010.

2. Rojas Cabrera E. Posibilidades de monitoreo del derecho a la salud en los primeros años de vida en Argentina: Una lectura a partir del acceso a las prestaciones y servicios comprometidos. En: Martinez Pizarro J, Rojas Cabrera E, Santillan Pizarro M, (ed.). El Consenso de Montevideo y la agenda de investigación sobre población y desarrollo: algunos temas seleccionados. Río de Janeiro: Asociación Latinoamericana de Población (ALAP); 2016. p. 25-44.

3. Organización Mundial de la Salud. Estadísticas Sanitarias Mundiales. Ginebra: OMS; 2009.

4. Organización Mundial de la Salud. Informe sobre la salud en el mundo 2008: La atención primaria de salud, más necesaria que nunca. Ginebra: OMS; 2008.
5. Organización Mundial de la Salud. Informe sobre la salud en el mundo 2000: Mejorar el desempeño de los sistemas de salud. Ginebra: OMS; 2000.

6. González Pons S. Sistema de monitoreo del desempeño del sistema de salud: Informe final de consultoría presentado a Fundación Plenitud, dentro del proyecto Observatorio de la Salud. Santo Domingo: Fundación Penitud; 2011.

7. Santillan Pizarro M, Ribotta B, Acosta L. Las posibilidades de las fuentes de información sociodemográficas para el monitoreo del derecho a la salud de las personas mayores en la Argentina, 1999-2013. En: Comisión Económica para América Latina y el Caribe. Notas de Población N 102, (LC/G.2673-P). Santiago: CEPAL; 2016. p 95-122.

8. Organización Mundial de la Salud, Health metrics network. Assessing the National Health information system: an assessment tool. Geneva: WHO; 2008.

9. Marracino C, Vera Figueroa M, Abadie JP. Indicadores de resultados para monitoreo de programas de coberturas [Internet]. 2001 [citado 10 may 2016]. Disponible en: http://tinyurl.com/ lbvq6uz. 
10. Consejo Nacional de Evaluación de la Política de Desarrollo Social. Indicadores de acceso y uso efectivo de los servicios de salud de afiliados al Seguro Popular. México DF: CONEVAL; 2014.

11. Huerta R, Paúl I, Leyton C. Impacto de indicadores de gestión en salud sobre estrategias de un servicio de salud público. Revista de Salud Pública. 2012;14(2):248-259.
12. Organización Panamericana de la Salud. Capacidades en salud pública en América Latina y el Caribe: evaluación y fortalecimiento. Washington DC: OPS; 2007.

13. Organización Mundial de la Salud. Monitoreo, evaluación y análisis de las estrategias nacionales de salud: Una plataforma dirigida por los países para la información y la rendición de cuentas. Ginebra: OMS; 2012.

\section{FORMA DE CITAR}

Peranovich AC. Capacidades y retos del monitoreo sobre la disponibilidad y el acceso a los servicios de salud en Argentina. 2017;13(1):73-90. doi: 10.18294/sc.2017.991.

Recibido: 20 de mayo de 2016 | Versión final: 27 de enero de 2017 | Aprobado: 2 de marzo de 2017

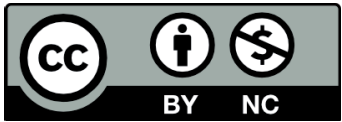

Este obra está bajo una licencia de Creative Commons Reconocimiento-NoComercial 4.0 Internacional. Reconocimiento - Permite copiar, distribuir y comunicar públicamente la obra. A cambio, se debe reconocer y citar al autor original. No Comercial - Esta obra no puede ser utilizada con finalidades comerciales, a menos que se obtenga el permiso. 